\title{
ON THE SOLUTION SETS OF DIFFERENTIAL INCLUSION IN BANACH SPACES
}

\author{
A. ANGURAJ AND K. BALACHANDRAN
}

A bstract. We prove the set of all classical solutions of the differential inclusion

$$
\begin{aligned}
& \dot{x}(t) \in A(t, x)+F(t, x, \dot{x}) \\
& x\left(t_{0}\right)=x_{0}, \quad \dot{x}\left(t_{0}\right)=y_{0}
\end{aligned}
$$

is a retract of the space $C^{1}$.

\section{Introduction.}

Let $I \subset R$ be a compact interval; $E$ a Banach space, $F$ a multifunction from $I \times E \times E$ into the subsets of $E ; A$ a continuous functions from $I \times E$ into $E$.

Given $t_{0} \in I, x_{0} \in E, y_{0} \in A\left(t_{0}, x_{0}\right)+F\left(t_{0}, x_{0}, y_{0}\right)$ consider the problem

$$
\begin{aligned}
& \dot{x} \in A(t, x)+F(t, x, \dot{x}) \\
& x\left(t_{0}\right)=x_{0} \\
& \dot{x}\left(t_{0}\right)=y_{0}
\end{aligned}
$$

A functions $\phi: I \rightarrow E$ is said to be a classical solution of (1) if $\phi \in C^{1}$ and $\dot{\phi}(t) \in A(t, \phi(t))+F(t, \phi(t), \dot{\phi}(t))$ for all $t \in I, \phi\left(t_{0}\right)=x_{0}, \dot{\phi}\left(t_{0}\right)=y_{0}$.

In this paper we prove that under suitable assumptions the set of all classical solutions of (1), $\Gamma\left(t_{0}, x_{0}, y_{0}, F\right)$ is a retract of $C^{1}$ which depends in a Lipschitzian

Received September 2, 1991. 
way on $x_{0}, y_{0}, F$. This is also true for the solution set of $\dot{x} \in A(t, x)+F(t, x, \dot{x})$,. $x\left(t_{0}\right)=x_{0}$. In [7] Ricceri discussed the problem without the term $A(t, x)$. Here our assumptions do not imply the compactness of $\Gamma\left(t_{0}, x_{0}, y_{0}, F\right)$ not even in $C$. Our result is different from many other recent works [1-3,5].

\section{Preliminaries and Notations.}

Let $X, Y$ be two nonempty sets. A multifunction $\Phi$ from $X$ into $Y(\Phi: X \rightarrow$ $\left.2^{Y}\right)$ is a function from $X$ into the family of all nonempty subsets of $Y$. When $X, Y$ are two topological spaces, we say that $\Phi$ is lower (upper) semicontinous if for every open (closed) set $\Omega \subset Y$, the set $\{x \in X: \Phi(x) \cap \Omega \neq \phi\}$ is open (closed) in $X$. A single valued function $f: X \rightarrow Y$ is said to be a selection of $\Phi$ if $f(x) \in \Phi(x)$ for all $x \in X$.

If $(\Sigma, \delta)$ is a metric space, for every $x \in \Sigma$ and nonempty $A, B \subset \Sigma$ we put

$$
\begin{aligned}
\delta(x, A) & =\inf _{z \in A} \delta(x, z) ; \delta^{*}(A, B)=\sup _{z \in A} \delta(z, B) \\
\delta_{H}(A, B) & =\max \left\{\delta^{*}(A, B), \delta^{*}(B, A)\right\} .
\end{aligned}
$$

Let $(X, d),(Y, \rho)$ be two metric spaces. A multifunction $\Phi: X \rightarrow 2^{Y}$ is said to be Lipschitzian if there exists a real number $L \geq 0$ (Lipschitz constant) such that

$$
\rho_{H}(\Phi(x), \Phi(z)) \leq \operatorname{Ldl}(x, z)
$$

for all $x, z \in X$. If $L<1$ we say that $\Phi$ is a multivalued contraction. Observe that any Lipschitzian multifunction is lower semicontinuous.

If $I$ is a compact real interval and $(E,\|\cdot\|)$ is a real Banach space, we denote by $C$ the space of all continuous functions from $I$ into $E$, equipped with the norm

$$
\|\phi\|_{C}=\max _{t \in I}\|\phi(t)\|
$$

We dentoe by $C^{1}$ the space of all continuously (strongly) differentiable functions from $I$ into $E$, equipped with the norm

$$
\|\phi\|_{C^{1}}=\|\phi\|_{C}+\|\ddot{\phi}\|_{C}
$$


where $\dot{\phi}$ is the (strong) derivative of $\phi$.

A set $A$ in a topological space $X$ is said to be a retract of $X$ if $A \neq \emptyset$ and there exists a continuous function $P: X \rightarrow A$ such that $P(x)=x$ for all $x \in A$.

\section{Main Result}

Now $(E,\|\cdot\|)$ is a real Banach space; $d$ is the metric induced by $\|\cdot\| . I$ is a (non-degenerate) compact real interval. Let $\alpha, \beta, \gamma: I \rightarrow[0, \infty)$ be continuous functions such that $\beta(t)<1, \forall t \in I$. Denote $\mathcal{L}_{\alpha, \beta, E}$ be the family of all lower semicontinuous multifunctions $F: I \times E \times E \rightarrow 2^{E}$, with closed and convex values such that for every $t \in I, x, y, u, v \in E$ one has

$$
d_{I H}(F(t, x, y), F(t, u, v)) \leq \alpha(t)\|x-u\|+\beta(t)\|y-v\|
$$

Let $A: I \times E \rightarrow E$ be a continuous function satisfying for every $x, y \in E, t \in I$,

$$
d(A(t, x), A(t, y)) \leq \gamma(t)\|x-y\|
$$

Let $t_{0} \in I, x_{0} \in E, F: I \times E \times E \rightarrow 2^{E}, A: I \times E \rightarrow E$. Then put

$$
\begin{gathered}
\Gamma\left(t_{0}, x_{0}, F\right)=\left\{\phi \in C^{1}: \phi(t)\right. \\
\text { for all } \left.t \in A(t, \phi(t))+F(t, \phi(t), \dot{\phi}(t))=x_{0}\right\}
\end{gathered}
$$

Further if $y_{0} \in A\left(t_{0}, x_{0}\right)+F\left(t_{0}, x_{0}, y_{0}\right)$ we put

$$
\Gamma\left(t_{0}, x_{0}, y_{0}, F\right)=\left\{\phi \in \Gamma\left(t_{0}, x_{0}, F\right): \dot{\phi}\left(t_{0}\right)=y_{0}\right\}
$$

Before stating our theorem, we recall a lemma which will be applied in the theorem. It follows at once from [1] and [2].

Lemma. Let $X$ be a paracompact topological space; $\left(Y,\|\cdot\|_{Y}\right)$ a Banach space; $\Phi: X \rightarrow 2^{Y}$ a lower semicontinuous mullifunction with closed and convex values; $f: X \rightarrow Y$ a continuous function; $\beta: X \rightarrow[0, \infty)$ a lower semicontinuous funclion such that $\delta(f(x), \Phi(x)) \leq \beta(x)$ for all $x \in X$, where $\delta$ is the metric 
induced by $\|\cdot\|_{Y}$. Then for every $\varepsilon>0$, there exists a continuous selection $g$ of. $\Phi$ such that $\|g(x)-f(x)\|_{Y} \leq \beta(x)+\varepsilon$ for all $x \in X$.

Theorem. Let $F \in \mathcal{L}_{\alpha, \beta, E}$. Then for every $t_{0} \in I, x_{0} \in E, y_{0} \in A\left(t_{0}, x_{0}\right)+$ $F\left(t_{0}, x_{0}, y_{0}\right)$, each of the sets $\Gamma\left(t_{0}, x_{0}, F\right), \Gamma\left(t_{0}, x_{0}, y_{0}, F\right)$ is a retract of the space $C^{1}$. Further if $F$ is a singlevalued function, then the set $\Gamma\left(t_{0}, x_{0}, F\right)$ is singleton.

Proof. Fix $t_{0} \in I, x_{0} \in E, y_{0} \in A\left(t_{0}, x_{0}\right)+F\left(t_{0}, x_{0}, y_{0}\right)$ and $L \in\left(\frac{1}{1-\beta^{*}}, \infty\right)$ with $\max _{t \in I} \beta(t)=\beta^{*} \in[0,1)$.

For each $\phi \in C$ put

$$
\|\phi\|_{0}=\max _{t \in I} e^{-L[\gamma(t)+\alpha(t)]\left|t-t_{0}\right|}\|\phi(t)\|
$$

Hence $\|\cdot\|_{0}$ is norm equivalent to $\|\cdot\|_{C}$.

Next, for every $\Psi \in C$ put

$$
\begin{aligned}
\Phi\left(x_{0}, \Psi, F\right)= & \left\{\phi \in C ; \phi(t) \in A\left(t, x_{0}+\int_{t_{0}}^{t} \Psi(\tau) d \tau\right)\right. \\
& \left.+F\left(t, x_{0}+\int_{t_{0}}^{t} \Psi(\tau) d \tau, \Psi(t)\right) \quad \text { for all } t \in I\right\}
\end{aligned}
$$

Here $\int_{t_{0}}^{t} \Psi(\tau) d \tau$ is Riemann integral.

Put $C_{y_{0}}=\left\{\phi \in C ; \phi\left(t_{0}\right)=y_{0}\right\}$ as.well as

$$
\Phi\left(x_{0}, y_{0}, \Psi, F\right)=\Phi\left(x_{0}, \Psi, F\right) \cap C_{y_{0}} \text { for each } \Psi \in C_{y_{0}}
$$

From [4], it follows that $\Phi\left(x_{0}, \Psi, F\right) \neq \emptyset$ for all $\Psi \in C$ and that $\Phi\left(x_{0}, y_{0}, \Psi, F\right) \neq$ $\emptyset$ for all $\Psi \in C_{y_{0}}$.

We now claim that the multifunctions $\Phi\left(x_{0}, \cdot, F\right)$ and $\Phi\left(x_{0}, y_{0}, \cdot, F\right)$ are multi-valued contractions with respect to the metric, say $\rho$ induced by $\|\cdot\|_{0}$ with Lipschitz constant $\frac{1}{L}+\beta^{*}$.

We prove this only for $\Phi\left(x_{0}, y_{0}, \cdot, F\right)$ since the proof for $\Phi\left(x_{0}, \cdot, F\right)$ is completely similar. 
$\operatorname{Fix} \Psi, \omega \in C_{y_{0}}, \phi \in \Phi\left(x_{0}, y_{0}, \Psi, F\right)$. Then for every $t \in I$, we have

$$
\begin{aligned}
& d\left(\phi(t), A\left(t, x_{0}+\int_{t_{0}}^{t} \omega(\tau) d \tau\right)+F\left(t, x_{0}+\int_{t}^{t_{0}} \omega(\tau) d \tau, \omega(t)\right)\right) \\
& \leq \gamma(t)\left\|\int_{t_{0}}^{t}(\Psi(\tau)-\omega(\tau)) d \tau\right\|+\alpha(t)\left\|\int_{t_{0}}^{t}(\Psi(\tau)-\omega(\tau)) d \tau\right\| \\
& \quad+\beta(t)\|\Psi(t)-\omega(t)\|
\end{aligned}
$$

Define a multifunction $H: I \rightarrow 2^{E}$ by

$$
H(t)= \begin{cases}A\left(t, x_{0}+\int_{t_{0}}^{t} \omega(\tau) d \tau\right)+F\left(t, x_{0}+\int_{t_{0}}^{t} \omega(\tau) d \tau, \omega(t)\right) & \text { if } t \neq t_{0}, t \in I \\ \left\{y_{0}\right\} & \text { if } t=t_{0}\end{cases}
$$

Then clearly $H$ is lower semicontinuous [4]. By Lemma, for every $\varepsilon>0, H$ admits a continuous selection $h$ such that

$$
\begin{aligned}
& \quad\|h(t)-\phi(t)\| \\
& \leq \gamma(t)\left\|\int_{t_{0}}^{t}(\Psi(\tau)-\omega(\tau)) d \tau\right\|+\alpha(t)\left\|\int_{t_{0}}^{t}(\Psi(\tau)-\omega(\tau)) d \tau\right\| \\
& \quad+\beta(t)\|\Psi(\tau)-\omega(\tau)\|+\varepsilon \\
& \left.\leq[\gamma(t)+\alpha(t)] \| \int_{t_{0}}^{t} \Psi(\tau)-\omega(\tau)\right) d \tau\|+\beta(t)\| \Psi(\tau)-\omega(\tau) \|+\varepsilon
\end{aligned}
$$

for every $t \in I$. In particular observe that $h \in \Phi\left(x_{0}, y_{0}, \omega, F\right)$.

Now we evaluate $\|h-\phi\|_{0}$. Assume $a<t_{0}$ and for every $t \in\left[a, t_{0}\right]$, we have

$$
\begin{aligned}
& e^{-L[\gamma(t)+\alpha(t)]\left(t_{0}-t\right)}\left\|\int_{t_{0}}^{t}(\Psi(\tau)-\omega(\tau)) d \tau\right\| \\
= & \| \int_{t_{0}}^{t} e^{-L[\gamma(t)+\alpha(t)](\tau-t)} \cdot c^{-L[\gamma(t)+\alpha(t)]\left(t_{0}-\tau\right)} \\
& (\Psi(\tau)-\omega(\tau)) d \tau \| \\
\leq & \|\Psi-\omega\|_{0} \int_{t}^{t_{0}} e^{-L[\gamma(t)+\alpha(t)](\tau-t)} d \tau \\
\leq & \frac{1}{L[\gamma(t)+\alpha(t)]}\|\Psi-\omega\|_{0}
\end{aligned}
$$

Therefore from (2) we have

$$
\begin{gathered}
e^{-L[\gamma(t)+\alpha(t)]\left(t_{0}-t\right)}\|h(t)-\phi(t)\| \leq\left[\frac{1}{L}+\beta(t)\right]\|\Psi-\omega\|_{0}+\varepsilon \\
\leq \leq\left(\frac{1}{L}+\beta^{*}\right)\|\Psi-\omega\|_{0}+\varepsilon
\end{gathered}
$$


Analogously if $t_{0}<b$ for every $t \in\left[t_{0}, b\right]$, we obtain

$$
e^{-L[\gamma(t)+\alpha(t)]\left(t-t_{0}\right)}\|h(t)-\phi(t)\| \leq\left(\frac{1}{L}+\beta^{*}\right)\|\Psi-\omega\|_{0}+\varepsilon
$$

Hence,

$$
\|h-\phi\|_{0} \leq\left(\frac{1}{L}+\beta^{*}\right)\|\Psi-\omega\|_{0}+\varepsilon
$$

Therefore

$$
\rho^{*}\left(\Phi\left(x_{0}, y_{0}, \Psi, F\right), \Phi\left(x_{0}, y_{0}, \omega, F\right)\right) \leq\left(\frac{1}{L}+\beta^{*}\right)\|\Psi-\omega\|_{0}
$$

Interchanging the roles of $\Psi$ and $\omega$ we get

$$
\rho^{*}\left(\Phi\left(x_{0}, y_{0}, \omega, F\right), \Phi\left(x_{0}, y_{0}, \Psi, F\right)\right) \leq\left(\frac{1}{L}+\beta^{*}\right)\|\Psi-\omega\|_{0}
$$

Since $\frac{1}{L}+\beta^{*}<1, \Phi\left(x_{0}, y_{0}, \cdot, F\right)$ is multivalued contraction. Similarly we can show that $\Phi\left(x_{0}, \cdot, F\right)$ is also multivalued contraction.

Now put

$$
P\left(x_{0}, F\right)=\left\{\phi \in C ; \phi \in \Phi\left(x_{0}, \phi, F\right)\right\}
$$

as well as

$$
P\left(x_{0}, y_{0}, F\right)=\left\{\phi \in C_{y_{0}} ; \phi \in \Phi\left(x_{0}, y_{0}, \phi, F\right)\right\}
$$

Then taking into account that all the sets $\Phi\left(x_{0}, \Psi, F\right), \Phi\left(x_{0}, y_{0}, \Psi, F\right)$ are convex closed, by Theorem 1 of [6] each of the sets $P\left(x_{0}, F\right), P\left(x_{0}, y_{0}, F\right)$ is a retract of the space $C$. In particular to see this for $P\left(x_{0}, y_{0}, F\right)$, take into account that $C_{y_{0}}$ being closed and convex, is in turn a retract of $C$.

Next consider the operator $T: C \rightarrow C^{1}$ defined by $T(\Psi)(t)=\int_{t_{0}}^{t} \Psi(\tau) d \tau$ for every $\Psi \in C, t \in I$. Clearly

$$
\begin{aligned}
\Gamma\left(t_{0}, x_{0}, F\right) & =\phi^{\left(x_{0}\right)}+T\left(P\left(x_{0}, F\right)\right) \\
\Gamma\left(t_{0}, x_{0}, y_{0}, F\right) . & =\phi^{\left(x_{0}\right)}+T\left(P\left(x_{0}, y_{0} ; F\right)\right)
\end{aligned}
$$

where $\phi^{\left(\dot{x_{0}}\right)}$ is the constant function on $I$ taking the value $x_{0}$. 
Let $\theta_{E}$ be the null element of $E$. Put

$$
V_{0}=\left\{\phi \in C^{1}: \phi\left(t_{0}\right)=\theta_{E}\right\}
$$

Clearly the operator $T$ is a linear homeomorphism from $C$ onto $V_{0}$. Therefore, each of the sets $T\left(P\left(x_{0}, F\right)\right), T\left(P\left(x_{0}, y_{0}, F\right)\right)$ is a retract of $V_{0}$. But $V_{0}$, being closed and convex, is a retract of $C^{1}$ and hence each of set $T\left(P\left(x_{0}, F\right)\right.$ ), $T\left(P\left(x_{0}, y_{0}, F\right)\right)$ is a retract of $C^{1}$. Hence from (3) and (4) $\Gamma\left(t_{0}, x_{0}, F\right)$ and $\Gamma\left(t_{0}, x_{0}, y_{0}, F\right)$ are retracts of $C^{1}$.

Further if $F$ is a single valued funtion, then from the classical contraction mapping principle of Banach-Caccioppoli, the set $\Gamma\left(t_{0}, x_{0}, F\right)$ is a singleton.

Hence the proof is completed.

\section{References}

[1] T. P. Aubin and A. Cellina, "Differential Inclusions", Springer-Verlag (1984).

[2] L. Gorniewicz, "On the solution sets of differential inclusions", J. Math. Anal. Appl., 113 (1986) 235-244.

[3] C. Himmelberg and F. Van Vleck, "A note on the solution sets of differential inclusions", Rocky Mountain J. Math., 12 (1982) 621-625.

[4] E. Michael, "Continuous selections I", Ann. of Math., 63 (1956) 361-382.

[5] N. S. Papageorgiou, "On multivalued evolution equations and differential inclusions in Banach spaces", Comment. Math. Univ. Sancli Pauli, 36 (1987), 21-39.

[6] B. Ricceri, "Une propriete topologigue de l'ensemble des points fixes d'une contraction multivoque a valeures convexes", Atti Accad. Naz. Lincei Rend. Cl. Sci. Fis. Mat. Natur., 81 (1987) 283-286.

[7] O. N. Ricceri, Classical solutions of the problcun $x^{\prime} \in F\left(t, x, x^{\prime}\right), x\left(t_{0}\right)=x_{0}, x^{\prime}\left(t_{0}\right)=y_{0}$ in Banach spaces, Funkcial. Ekvac. 34 (1991) 127-141.

Department of Mathematics, Bharathiar University, Coimbatore 641 046, Tamil Nadu India. 